\title{
Clinical and pathological features of primary renal angiosarcoma
}

\author{
Roberto lacovelli, MD; Valentina Orlando, MD; Antonella Palazzo, MD; Enrico Cortesi, MD
}

Sapienza University of Rome; Department of Radiology, Oncology and Human Pathology, Oncology Unit B, Rome, Italy

Cite as: Can Urol Assoc J 2014;8(3-4):e223-6. http://dx.doi.org/10.5489/cuaj.1585

Published online April 14, 2014.

\section{Abstract}

Introduction: We review all cases of renal angiosarcoma described in the medical literature to describe its clinical and histological features.

Methods: The search term was "angiosarcoma of the kidney." All articles or case reports in English, French, German or Spanish published until March 15, 2013 were considered. When available, data that focused on clinical and pathological features were extracted. Results: In total, the final cohort included 42 cases. The median overall survival (OS) was 5.0 months (95\% confidence interval [CI], 2.1-7.9). Patients with metastatic disease had more than a threefold increase in the risk of death compared to patients without metastatic disease (hazard ratio: $3.27,95 \% \mathrm{Cl}, 1.48-7.24 ; p=0.004$ ). Non-metastatic patients had dismal disease-free survival (DFS) rates, with a median DFS of 6.0 months $(95 \% \mathrm{Cl}, 4.3-7.7)$; despite this, chemotherapy was effective to increase survival in eligible patients (4.0 vs. 1.0 months; $p=0.001)$. Microscopic examination found epithelioid and spindled cell in $44 \%$ and $56 \%$ of cases, respectively; there was a statistically insignificant increase in survival in patients with epithelioid patterns compared to spindled ones (9.0 vs. 4.0 months; $p=0.077$ ). The tumour grading was related to OS; the lower the grade, the longer the survival (13 vs. 4 months; $p=0.029$ ).

Conclusions: Renal angiosarcoma is a rare disease with defined clinical and pathological features. It has a very poor prognosis in patients with metastatic disease and a high recurrence rate in patients with non-metastatic disease.

\section{Introduction}

Angiosarcoma is a highly aggressive tumour consisting of vascular spaces lined by malignant endothelial cells, and it represents $2 \%$ of all soft-tissue sarcomas. Angiosarcoma is generally located in the skin, superficial soft tissue or other organs, such as breast, liver or bone. ${ }^{1}$ Its primary occurrence in the kidney is extremely rare and generally characterized by a poor prognosis because of its rapid local and bloodstream dissemination. $^{2}$

To date, the typical risk factors for angiosarcoma, such as arsenic, thorotrast, vinyl chloride and radiation, have not been associated with renal localization. Smoking or androgens could explain its predominance in males, but there is no data to support this association. ${ }^{3}$ In fact, renal angiosarcoma seems to be more frequent in older men with nonspecific clinical presentation characterized by flank pain and hematuria, mimicking other renal malignancies. ${ }^{4}$

Because of its rarity, there is no standard therapy. Surgery is generally the first step, while subsequent therapies are varied and not well-defined. In this study, we review all published cases of renal angiosarcoma in order to describe its clinical and histological features.

\section{Methods}

As previously described, ,5,6 a systematic search in PubMed and Scopus was performed to identify all articles describing patients with primary angiosarcoma of the kidney. The search term was "angiosarcoma of the kidney." We considered all articles or case reports in English, French, German or Spanish published until March 15, 2013. References of selected articles were also investigated and, if adequate, included for final analysis.

When available, data focusing on clinical and pathological features were extracted. Clinical data included age, sex, presence and type of symptoms, stage of disease at diagnosis, sites of metastases, disease-free survival (DFS), overall survival (OS), progression-free survival (PFS), and type of treatment for renal angiosarcoma and its efficacy. The pathological features included maximum extension of primary tumour measured (in centimetres), and the tumour grade of differentiation defined as "low grade" for grade 1 or 2 and "high grade" for grade 3 or 4 . The immunohistochemical expression (yes/no) of several markers was evaluated; these were vimentin, cytokeratin (CK), CD117, CD68, CD45, CD34, CD31, CD10, epithelial membrane 
Iacovelli et al.

antigen (EMA), desmin, smooth muscle actin (SMA), Factor VIII, S100, Ulex europaeus agglutinin, I (UEA), carcinoembryonic antigen (CEA), actin, melan-A, HMB45 and vascular endothelial growth factor (VEGF). All data were used to create a database for final analysis.

\section{Statistical analysis}

Baseline values were expressed as median and interquartile range (IQR), with baseline defined as the date of surgical diagnosis. DFS was defined as the length of time after surgery during which no disease was found. The OS was defined as the time from surgery to death or last contact. Finally, the PFS was defined as the time from the start of therapy to the progression of disease or the death for any cause. The DFS, PFS and OS were estimated using Kaplan-Meier method with $95 \%$ confidence intervals $(\mathrm{Cl})$ and compared across the groups using the log-rank test.

The correlations between tumour extension and histological features were evaluated by the non-parametric Spearman rank test. The chi-square test was used to assess the differences between groups.

Association of extension of disease at diagnosis and OS were evaluated using the Cox proportional hazards model. All variables were considered significant at $p<0.05$. All statistical analyses were performed using the Predictive Analytics SoftWare (PASW, v.19; IBM SPSS Inc.).

\section{Results}

\section{Clinical features}

The final cohort included 42 cases (Table 1); most were male (86\%), and the median patient age was 60 (IQR: 49-71.5).

Most patients $(85.4 \%)$ had clinical symptoms as first presentation of disease; pain was the most frequent symptom $(74 \%)$, followed by hematuria $(62 \%)$, anemia (33\%) and renal mass $(27 \%)$, while hemoptysis, fever and weight decrease were each reported in about $10 \%$ of cases.

Most patients (93\%) had had a radical nephrectomy as treatment for their primary tumour. One-third of patients $(33 \%)$ were metastatic at diagnosis and two-thirds of nonmetastatic patients developed metastasis after tumour extirpation. Data about DFS were available in 21 cases and showed a median DFS of 6.0 months $(95 \% \mathrm{Cl}, 4.3-7.7)$.

Half of the metastatic patients had 2 or more sites of metastases. These sites included lung (46\%), liver $(46 \%)$, bone $(39 \%)$, local relapse $(19 \%)$, and abdominal lymphnodes $(11 \%)$. Soft tissues, skin, bowels, spleen and peritoneum were reported in less than $5 \%$ of cases.

In about 11 patients treated with first-line chemotherapy, the median PFS was 2.0 months $(95 \% \mathrm{Cl}, 1.0-3.0)$, with

\begin{tabular}{|c|c|c|}
\hline Author & $\begin{array}{l}\text { PubMed ID } \\
\text { or reference number }\end{array}$ & No. cases \\
\hline Adijman S et al. & 2401309 & 1 \\
\hline Akkad T et al. & 16903939 & 1 \\
\hline Aksoy $Y$ et al. & 11803270 & 1 \\
\hline Allred CD et al. & 7196879 & 1 \\
\hline Askari A et al. & 7005464 & 1 \\
\hline Aydogdu I et al. & 10438072 & 1 \\
\hline Berretta $\mathrm{M}$ et al. & 16736893 & 1 \\
\hline carnero Lòpez B et al. & 18158986 & 1 \\
\hline Cason JD et al. & 3629777 & 1 \\
\hline Cerilli LA et al. & 9786357 & 1 \\
\hline Costero-Barrios CB et al. & 15456157 & 1 \\
\hline Desai MB et al. & 2734970 & 1 \\
\hline Douard A et al. & 22657265 & 1 \\
\hline Fukunaga M. & 19147300 & 1 \\
\hline Garmendia JC et al. & 2763892 & 1 \\
\hline Hiratsuka $Y$ et al. & 9179675 & 1 \\
\hline Johnson VV et al. & 11900578 & 1 \\
\hline Juan CJ et al. & Ref. 8 & 1 \\
\hline Kern SB et al. & 7802558 & 2 \\
\hline Kragel PJ et al. & 1413363 & 1 \\
\hline Leggio L et al. & 16818182 & 1 \\
\hline Li $\mathrm{N}$ et al. & 10374567 & 1 \\
\hline Limmer $\mathrm{S}$ et al. & 11572116 & 1 \\
\hline Lopez Cubillana P et al. & 15270285 & 1 \\
\hline Martinez-Pineiro L et al. & 7618041 & 1 \\
\hline Matter LE et al. & 10081105 & 1 \\
\hline Mordkin RM et al. & 9386065 & 1 \\
\hline Nguyen T et al. & 21242919 & 1 \\
\hline Peters $\mathrm{HJ}$ et al. & 4548091 & 1 \\
\hline Prince CGL & Ref. 9 & 1 \\
\hline Sanyal B et al. & 574526 & 1 \\
\hline Singh $\mathrm{C}$ et al. & 21698784 & 1 \\
\hline Souza OE et al. & 16953913 & 1 \\
\hline Terris $\mathrm{D}$ et al. & 3740062 & 1 \\
\hline Testa G et al. & Ref. 10 & 1 \\
\hline Tsuda $\mathrm{N}$ et al. & 9413038 & 1 \\
\hline Witczak W et al. & 8170817 & 1 \\
\hline Yamamoto $\mathrm{Y}$ et al. & 16617877 & 1 \\
\hline Yau T et al. & 17571263 & 1 \\
\hline Yoshida $\mathrm{K}$ et al. & 21139907 & 1 \\
\hline Zenico T et al. & 21989448 & 1 \\
\hline
\end{tabular}

disease control (response or stability) in $44 \%$ of cases. The median OS was greater in patients treated with chemotherapy compared to the untreated (4.0 vs. 1.0 months; $p=0.001$ ). As for adjuvant setting, combined chemotherapy was the most preferred approach, and the common medications were anthracyclines, ifosfamide and dacarbazine; only 1 patient was treated with anti-angiogenic drugs sunitinib with a PFS of 9 months. 


\begin{tabular}{|c|c|c|c|c|c|c|c|c|}
\hline Factor & Vimentine & CKs & CD31 & CD34 & Desmin & Fact VIII & S100 & HMB45 \\
\hline No. cases & 11 & 16 & 15 & 16 & 5 & 19 & 8 & 6 \\
\hline Positive staining & $73 \%$ & $13 \%$ & $100 \%$ & $94 \%$ & $0 \%$ & $79 \%$ & $0 \%$ & $0 \%$ \\
\hline \multicolumn{9}{|l|}{ Histology } \\
\hline Epithelioid & $50 \%$ & $25 \%$ & $100 \%$ & $100 \%$ & - & $83 \%$ & $0 \%$ & $0 \%$ \\
\hline Spindle & $86 \%$ & $0 \%$ & $100 \%$ & $83 \%$ & $0 \%$ & $75 \%$ & $0 \%$ & $0 \%$ \\
\hline
\end{tabular}

\section{Pathological features}

The median value for the longest tumour diameter was $13 \mathrm{~cm}$ (IQR: 9-19). No differences in terms of OS or DFS were deemed related ( $p=0.57$ and $p=0.79$, respectively) to the primary tumour extension above or under the median value.

Upon microscopic examination, we found epithelioid or spindled cell in $44 \%$ and $56 \%$ of cases, respectively. Even if a greater OS was found in patients with the epithelioid compared to the spindle pattern (9.0 vs. 4.0 months), this difference was not significant $(p=0.077)$. In analysing differentiation, we found high-grade tumours in $80 \%$ and low-grade tumours in $20 \%$ of cases. The tumour grade was related to OS; the lower the grade, the longer the survival (13.0 vs. 4.0 months; $p=0.029$ ) (Fig. 1).

We tallied the immunohistochemical expression of a variety of tested markers (Table 2). All markers were tested for primary tumour extension, grading, status of disease at diagnosis or among each other. Spearman's correlation did not find any direct relationship between the parameters reported above.

\section{Discussion}

This study is the first to report a clinical and pathological description of RA. Similarly, to other renal tumours, the initial diagnosis is made based on local symptoms, such as hematuria, anemia and renal mass. Even if we are unable to confirm the predictive/prognostic role of primary nephrectomy, it is reasonable to consider this surgical approach to palliate the tumour's related symptoms and, eventually, to perform a more exact diagnosis of this rare tumour.

Recently, some authors reported a case of renal angiosarcoma in which the diagnosis was performed by cytology analysis on an fine-needle aspiration (FNA) sample. ${ }^{7}$ They describe several cellular characteristics that may be useful for cytological diagnosis, and this may be an interesting approach to making a precocious diagnosis and eventually to start systemic therapy early in case of metastatic disease.

Since 1981, patients have been treated with different therapies based upon previous knowledge of other types of sarcomas, but considering the poor prognosis of this tumour, comparing these treatments to supportive care has never been assessed. ${ }^{8-10}$
Our results confirm the dismal prognosis of this disease. Patients have a short survival regardless of the presence of metastases at diagnosis. Moreover, several baseline characteristics seem to have a prognostic role: the advanced stage, the number of metastatic sites, the grade of tumour differentiation and a trend were also found in the epithelioid histology compared to the spindle cells. Although Leggio and colleagues reported a better outcome for patients with a primary tumour size less than $5 \mathrm{~cm}$, we did not find any differences in survival based on primary tumour extension. ${ }^{1}$

We found that some markers, such as cytokeratins (CKs) and CD31, are mainly expressed in the epithelioid subtype, while the spindle cell histology is principally characterized by a greater expression of vimentin and by a negative expression of desmin and cytokeratins. These data need to be confirmed in a larger dataset, but their clinical role should be kept in mind considering the different patient outcomes related to histology reported in our data.

Our study has some important limitations including its retrospective nature and the small number of patients valuable for each variable may increase the risk of bias. Furthermore, authors of different articles did not use the same criteria for evaluation, and the sensitivity of the diagnostic tech-

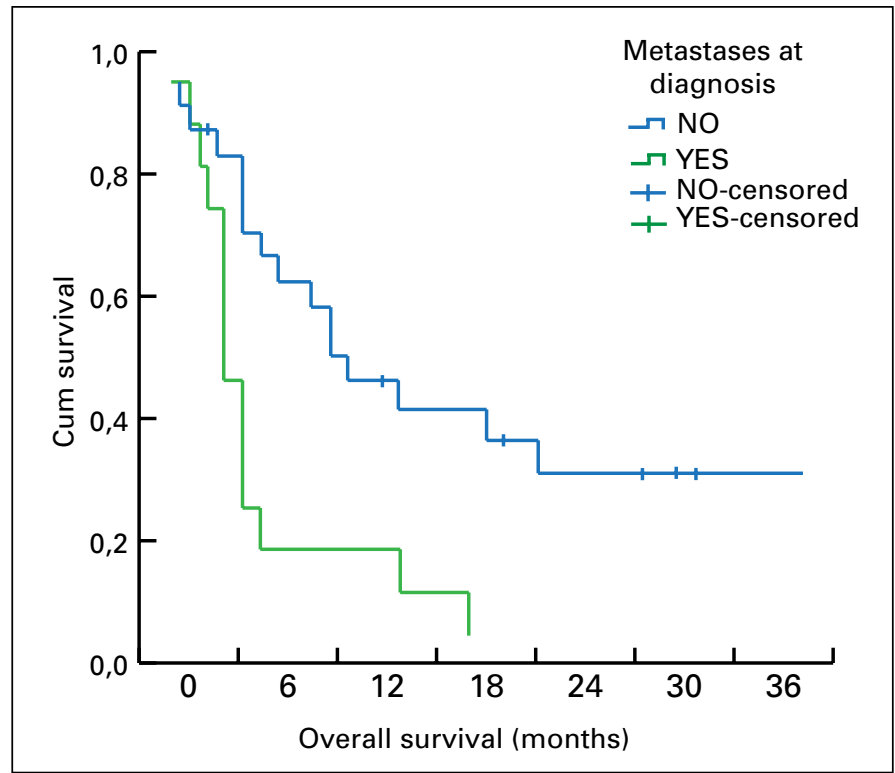

Fig. 1. Overall survival in patients affected by metastatic or non-metastatic renal angiosarcoma at diagnosis. 
Iacovelli et al.

niques has increased during recent years. Patients treated with chemotherapy received several different regimens and there were no uniform evaluation criteria at the time of discontinuation. In addition, published cases may not represent the general population, even though, as previously reported, ${ }^{5}$ the rarity of the disease may be itself a reason to submit a case report.

\section{Conclusion}

Renal angiosarcoma is a rare disease with a well-defined immunohistochemical pattern. It is clinically characterized by a very poor prognosis, mainly for patients with metastases at diagnosis, and by a high recurrence-rate in patients with localized disease. Even if chemotherapy may be recommended in eligible patients and the role of anti-angiogenic agents may be promising, this needs to be further evaluated.

Competing interests: Dr. lacovelli, Dr. Orlando, Dr. Palazzo and Dr. Cortesi all declare no competing financial or personal interests.

This paper has been peer-reviewed.

\section{References}

1. Leggio L, Addolorato G, Abenavoli L, et al. Primary renal angiosarcoma: A rare malignancy. A case report and review of the literature. Urol Oncol 2006;24:307-12. http://dx.doi.org/10.1016/i.urolonc.2005.10.002

2. Zenico T, Saccomanni M, Salomone U, et al. Primary renal angiosarcoma: Case report and review of world literature. Tumori 2011;97:e6-9.

3. Nguyen T, Auquier MA, Renard C, et al. Hemoptysis and spontaneous rupture of a primary renal angiosarcoma: A case report [article in French]. J Radiol 2010;91:1313-7. http://dx.doi.org/10.1016/ SO221-0363(10)70201-8

4. Kern SB, Gott L, Faulkner J. Occurrence of primary renal angiosarcoma in brothers. Arch Pathol Lab Med 1995;119:75-8

5. lacovelli R, Altavilla A, Ciardi A, et al. Clinical and pathological features of primary renal synovial sarcoma: Analysis of 64 cases from 11 years of medical literature. BJU Int 2012;110:1449-54. http://dx.doi. org/10.1111/j.1464-410X.2012.11105.x

6. Risi E, lacovelli R, Altavilla $A$, et al. Clinical and pathological features of primary neuroectodermal tumor/Ewing sarcoma of the kidney. Urology 2013;82:382-6. http://dx.doi.org/10.1016/i.urology.2013.04.015

7. Singh C, Xie L, Schmechel SC, et al. Epithelioid angiosarcoma of the kidney: A diagnostic dilemma in fineneedle aspiration cytology. Diagn Cytopathol 2012;40:E131-9. http://dx.doi.org/10.1002/dc.21762

8. Juan CJ, Wu CJ, Hsueh CJ, et al. Primary renal angiosarcoma: A case report. Chin J Radiol 2000;25:87-90.

9. Prince CHL. Primary angio-endothelioma of the kidney: Report of a case and brief review. J Urol 1942;112:723-6.

10. Testa $G$, Talamona $G$, Tufano $A$, et al. Primary renal angiosarcoma: $A$ case report. Acta Urologica Italica $1998 ; 12: 225-7$

Correspondence: Dr. Roberto lacovelli, Sapienza University of Rome; Department of Radiology, Oncology and Human Pathology, Oncology Unit B. Viale Regina Elena 324, 00161 Rome, Italy ; roberto.iacovelli@alice.it 